\title{
A utilização da ofurôterapia para recém-nascidos pré-termo hospitalizados
}

\author{
The use of ofurothery for hospitalized premost newborns \\ El uso de ofurotería para los recién nacidos hospitalizados
}

Raiane Santos de Brito ${ }^{1}$, Carla Lorena de Araújo Costa ${ }^{1}$, Luana de Jesus Reis ${ }^{1}$, Carolline Bittencourt da Cruz¹, Lília Tatiane dos Santos Sá1, Izadora Santos Paciência**.

\begin{abstract}
RESUMO
Objetivo: Explanar acerca dos benefícios que a ofurôterapia oferece ao recém-nascido pré-termo hospitalizado. Métodos: Revisão de literatura integrativa no período de 2009 a 2019 nas bases de dados Scielo, Medline, Pubmed, Google Acadêmico. O processo de análise dos artigos selecionados deu-se por meio da leitura exploratória e detalhada de títulos, resumos e dos resultados das pesquisas, onde se buscaram a utilização da ofurôterapia para recém-nascidos pré-termo hospitalizados. Resultados: A ofurôterapia através das propriedades físicas da água aliada com a temperatura, em geral entre $36^{\circ}$ e $37^{\circ} \mathrm{C}$, atua sobre as terminações nervosas do paciente limitando a condução dolorosa, além de agir diretamente nos sinais vitais, encaminhando-o para a homeostase. Alcançando resultados positivos como: sendo no ganho de peso corporal, melhora na qualidade sono, redução do quadro álgico ou estabilização dos sinais vitais, se mostrando um recurso valioso na assistência hospitalar ao recém-nascido pré-termo. Considerações finais: A ofurôterapia se mostrou efetiva, já que de maneira clara e objetiva observou-se a evolução dos pacientes de forma eficaz e positiva, concluindo, de tal modo, a importância da técnica durante o período de hospitalização.
\end{abstract}

Palavras-chave: Hidroterapia, Pediatria, Recém-nascido, Prematuro.

\begin{abstract}
Objective: Explain about the benefits that hot tub offers to the hospitalized preterm newborn. Methods: Literature review from 2009 to 2019 in the databases Scielo, Medline, Pubmed, Google Scholar. The analysis process of the selected article took place through the exploratory and detailed reading of titles, abstracts and research results, where the use of hot tub therapy for hospitalized preterm newborns was sought. Results: The hot tub therapy through the physical properties of water combined with the temperature in general between $36^{\circ}$ and $37^{\circ} \mathrm{C}$ acts on the patient's nerve endings limiting the painful conduction, besides acting directly on the vital signs leading him to homeostasis reaching different results in the patient. however positive, being in body weight gain, improvement in sleep quality, reduction in pain or stabilization of vital signs, proving to be a valuable resource in hospital care for preterm newborns. Conclusion: The hot therapy was effective, the authors discussed the indication of the technique, since clearly and objectively observed the evolution of patients from their research effectively and positively, thus concluding the importance of the technique in the period of hospitalization of these patients.
\end{abstract}

Key words: Hydrotherapy, Pediatrics, Newborns, Premature.

\section{RESUMEN}

Objetivo: Explique los beneficios que ofrece la bañera de hidromasaje para el recién nacido prematuro hospitalizado. Métodos: Revisión de literatura intregadora de 2009 a 2019 en las bases de datos Scielo, Medline, Pubmed, Google Scholar. El proceso de análisis de los artículos seleccionados llevó a cabo a través

${ }^{1}$ Centro Universitário UNIRB (FARAL), Alagoinhas-BA. *E-mail: raianebrito18.rb@gmail.com 
de la lectura exploratoria y detallada de títulos, resúmenes y resultados de investigación, donde se buscó el uso de la terapia de hidromasaje para recién nacidos prematuros hospitalizados. Resultados: La terapia de bañera de hidromasaje a través de las propiedades físicas del agua, combinadas con la temperatura, en general entre $36^{\circ}$ y $37^{\circ} \mathrm{C}$, actúa sobre las terminaciones nerviosas del paciente limitando la conducción dolorosa, además de actuar directamente sobre los signos vitales que lo llevan al homeostasis. alcanzando diferentes resultados en el paciente. Ganar peso corporal, mejorar la calidad del sueño, reducir el dolor o estabilizar los signos vitales. demostrando ser un recurso valioso en la atención hospitalaria para los recién nacidos prematuros. Conclusión: La terapia de calor fue efectiva ya quela evolución de los pacientes efectiva y positiva; concluyendo así la importancia de la técnica en el período de hospitalización

Palabras clave: Hidroterapia, Pediatría, Recién nacido, Prematuro.

\section{INTRODUÇÃO}

A prematuridade é definida como o nascimento que ocorre entre a vigésima e trigésima sétima semanas de gestação, caracterizada pela imaturidade do organismo, onde ainda não se realiza troca gasosa de modo efetivo, tão pouco se tem nutrição apropriada, devido sua baixa. Em virtude disso, os prematuros são submetidos a uma série de procedimentos que causam desconforto e estresse (SILVA H, et al., 2017).

Um dos principais comprometimentos que este paciente está exposto, devido a sua condição, é a imaturidade pulmonar, por conta da sua incapacidade na produção de surfactante, debilitando assim a insuflação alveolar. Em geral os recém-nascidos pré-termo nascem abaixo do peso ideal, além de desenvolverem possíveis comprometimentos neurológicos tais como hemorragias peri e intraventriculares e a leucomalácia periventricular.com isso existe a grande possibilidade que estes RNPT'S estão sujeitos ao déficit motor, cognitivo, neuropsicomotor e de linguagem. Em virtude desses possíveis cenários é necessária a assistência logo após o nascimento, com intenção de reparar o mais breve possível a homeostase (OLIVEIRA C, et al., 2015).

O Brasil está entre os 10 países do mundo com índices mais altos de prematuridade, alcançando uma marca de $60 \%$ de nascimentos prematuros. Somente no ano de 2010 foram registrados 1 milhão de óbitos diretamente ligados a prematuridade, 1 a cada 10 nascimentos deste mesmo ano foram prematuros; considerando que houveram cerca de 15 milhões de nascimentos, houve uma porcentagem de $11,1 \%$ nascimentos pré-termo (OLIVEIRA L, et al., 2016).

As causas espontâneas da prematuridade estão relacionadas a alguns fatores variados como: baixo nível socioeconômico, gestações que ocorrem em mulheres com idade abaixo de 21 anos ou com idade acima dos 36 anos. Além destas, a estatura materna abaixo de 1,52 metros de altura, histórico de parto prematuro, hemorragias vaginais estando ainda no $2^{\circ}$ semestre gestacional, infecções urinárias, amadurecimento cervical precoce e o aumento da atividade uterina antes da 29a semana gestacional (SALGE A, et al., 2013). Salge A, et al. (2013) afirma que apesar da prematuridade de modo espontâneo ela ainda pode ocorrer de modo eletivo, significando que o médico pode optar por realizar o parto antes da 37ª semana de gestação, prevenindo possível risco de vida para genitora ou feto; um exemplo dessa situação é em caso de gestação gemelar, ou seja, quando existem dois fetos.

Diante das necessidades que o prematuro apresenta é possível compreender a importância da hospitalização neonatal, que por sua vez trata-se de um ambiente terapêutico seguro e adequado para os cuidados do RNPT, contando com uma diversidade de aparelhos tecnológicos e profissionais especializados, com o constante propósito de ofertar um tratamento eficaz. Dentro deste ambiente se encontra a equipe multidisciplinar, composta por: médicos neonatologistas, enfermeiros, técnicos em enfermagem, fonoaudiólogos e fisioterapeutas (RIBEIRO J, et al., 2016).

Considerando o profissional fisioterapeuta dentro da hospitalização neonatal, destaca-se entre suas atribuições a atuação na redução de sequelas sistêmicas, proporcionando aumento da sobrevida do prematuro, além favorecer todo desenvolvimento cinético funcional em todo e qualquer sistema. Muito embora seja considerada recente sua participação na UTI neonatal, a fisioterapia mostra-se indispensável no 
favorecimento respiratório do RNPT e no desenvolvimento neuropsicomotor, possibilitando a diminuição no tempo de internação do prematuro na UTI neonatal (MAIA F, 2015).

Pinheiro E, et al. (2011) em seu estudo traz que o estresse tem como causa principal o ruído presente no ambiente da internação. Os sons dos equipamentos em geral e da rotina do local, unidos ao som da incubadora, e sons produzidos pelo próprio prematuro, reverberam por toda cúpula da incubadora. Tendo em mente que esse prematuro estava acostumando ao ambiente intrauterino, que possui uma barreira de som natural, fica latente o entendimento de toda agitação que é causada nele, esta é atrelada ao aumento da frequência cardíaca, respiratória e pressão arterial.

Diante do desconforto, dor e estresse sofrido pelo RNPT, existe a constante necessidade de procedimentos e técnicas que possam oferecer maior qualidade de vida e gerar homeostase. A fisioterapia vai apresentar uma série de recursos que atendam às necessidades deste prematuro, dentre eles existe a ofurôterpia, que está inserida na modalidade da fisioterapia aquática, também chamada de hidroterapia. no Brasil a hidroterapia começou a ser utilizada em 1922 na cidade do Rio de Janeiro, na Santa Casa de Misericórdia (ROUT A, 2000 apud TANTERFFER A, s.d.).

A ofurôterapia teve origem na Holanda em 1997, criada a partir do banho humanizado, por obstetras e enfermeiros. No Brasil é conhecido também como banho de ofurô, e vem sendo adotada nos ambientes hospitalares como uma conduta de humanização para o RNPT (GONSALVES R, et al., 2017). O procedimento, possui a necessidade de um ambiente calmo com a iluminação o mais baixo possível, de forma a favorecer o relaxamento, além de manter o controle da temperatura da água entre 36,5 e $37^{\circ} \mathrm{C}$. No que se refere aos equipamentos, são utilizados o termômetro para o monitoramento da temperatura, luvas para proteção do profissional e do RNPT, toalhas para contenção e preservação da temperatura corporal, além é claro, do balde de plástico de até 9 litros, dos quais são utilizados somente 6 litros, transparente para total visualização e segurança do prematuro.

Sempre que possível a terapia conta com a presença da genitora ou cuidador, preservando laços afetivos e a sociabilidade do prematuro (ATAÍDE V, et al., 2016). Sua contraindicação é feita para prematuros com peso abaixo de 1250 gramas, que se encontre em estado febril, que possua erupções cutâneas contagiosa, feridas abertas, infecções, hiper ou hipotensão não controlada e que possuam gastrotomia, suporte ventilatório, histórico de convulsões, histórico de doenças cardiológicas além de má formações ou lesões ortopédicas graves (GONSALVES R, et al., 2017). Diante do cenário exposto o questionamento norteador deste trabalho foi: Quais os benefícios que a ofurôterapia pode oferecer aos RNPT's hospitalizados?

A justificativa está pautada na grande incidência da prematuridade que há no país, e por se tratar de uma técnica relativamente nova, pouco conhecida e consequentemente pouco utilizada; desse modo existe a necessidade de gerar conhecimento ao público alvo (fisioterapeutas e acadêmicos em fisioterapia) e a sociedade como um todo. Afim de que obtenha maior visibilidade, gerando assim expansão de seu uso na área, favorecendo e otimizando os cuidados do paciente RNPT; quanto ao objetivo do trabalho, é explanar acerca dos benefícios que a ofurôterapia oferece ao RNPT hospitalizado.

\section{MÉTODOS}

O trabalho foi realizado através do método de revisão narrativa de literatura. A coleta de dado ocorreu de fevereiro de 2019 a outubro de 2019, foram utilizados artigos encontrados nas bases de dados como Scielo, Medline, Pubmed, Google Acadêmico. Quanto às variáveis, foram elas frequência cardíaca, frequência respiratória, sono e peso Para a escolha dos materiais utilizados na construção do trabalho foram usados os seguintes descritores: "hidroterapia", "UTI neonatal", "pré-termo", "prematuridade" e "ofurôterapia na UTI".

Para a construção desse deste trabalho foram utilizados 16 artigos que respeitaram os seguintes critérios de inclusão baseados na compatibilidade com o assunto tratado, o ano de publicação respeitando um prazo de validade de 10 anos, de modo que foram incluídos todos com data de publicação entre janeiro de 2009 a agosto de 2019, além de ter como idiomas o português ou inglês. Todos os artigos que não correspondiam aos critérios de inclusão foram excluídos. 


\section{RESULTADOS}

Silva $\mathrm{H}$, et al. (2017) construiu um estudo intitulado "Efeitos fisiológicos da hidroterapia em balde em recémnascidos prematuros", por que teve início em janeiro de 2015 e terminou em janeiro de 2016, sendo realizado na Unidade de Cuidados Intermediário do Hospital Regional do Mato Grosso do Sul, na cidade Campo grande. Para poder participar do estudo o paciente deveria atender aos seguintes critérios de inclusão: ter mais de 72 horas de vida, estar em ventilação espontânea em ar ambiente, hemodinamicamente estável, sem acesso periférico, com peso mínimo de 1500 gramas e com uma progressão na curva desse ganho diariamente, além de não apresentar estomias, má formação congênita e liberação médica para banho diário.

Inicialmente foram admitidos 50 pacientes, contudo 20 receberam alta ou os pais não prosseguiram no estudo, 30 dos que permaneceram tinham idade gestacional entre 27 a 35 semanas, 17 dos pacientes eram do sexo masculino e 13 do sexo feminino. O procedimento envolveu 2 sessões com duração de 10 minutos, em dias alternados, nos períodos matutino e vespertino, sendo $\mathrm{RN}$ imerso em água com temperatura de $36.5^{\circ}$ a $37^{\circ} \mathrm{C}$, até a altura das escápulas. A avaliação era feita 15 minutos antes do procedimento, imediatamente após o procedimento e a última avaliação 30 minutos após o procedimento; as variáveis da avaliação foram: frequência cardíaca $(F C)$, frequência respiratória $(F R)$, saturação de oxigênio $(\mathrm{SaO} 2)$ e peso.

Tabela 1 - Resultados encontrados na avalição do estudo de Silva H, et al., 2017.

\begin{tabular}{cccc}
\hline CF & $\mathbf{1 5}$ min antes & AV. imediata & AV. após 30 min \\
\hline Dia 1 & $152 \mathrm{bpm}$ & $154 \mathrm{bpm}$ & $146 \mathrm{bpm}$ \\
Dia 2 & $152 \mathrm{bpm}$ & $153 \mathrm{bpm}$ & $147 \mathrm{bpm}$ \\
FC & & & $55 \mathrm{ir}$ \\
Dia 1 & $55 \mathrm{ir}$ & $51 \mathrm{ir}$ & $55 \mathrm{ir}$ \\
Dia 2 & $53 \mathrm{ir}$ & $56 \mathrm{ir}$ & \\
\hline Saturação de O2 & & $97,2 \%$ \\
Dia 1 & $96,2 \%$ & $96,6 \%$ & $96,6 \%$ \\
Dia 2 & $96,6 \%$ & $97 \%$ &
\end{tabular}

Fonte: Brito RS, et al., 2020.

Dentre os resultados observados na avaliação, o mais significativo foi o ganho de peso, após a primeira sessão a média de peso aumentou cerca de 70 gramas e após a segunda sessão o ganho médio de peso foi de 55, 44 gramas. Por fim o estudo conclui que ofurôterpia é uma técnica terapêutica segura para o ganho de peso, a ação da água aquecida fornece conforto e relaxamento ao paciente. Rambo D e Filippin N, (2019) realizou um estudo intitulado como no hospital de médio porte na região nordeste do Rio Grande do Sul, na unidade de terapia intensiva "Efeitos da fisioterapia aquática em prematuros internados na UTI", em um hospital na regiãol de médio porte no nordeste do Rio Grande do Sul durante o mês de março no ano de 2018 o trabalho trata-se de um ensaio clinico randomizado do tipo series temporais.

Foram incluídos na pesquisa os RNPT's com quadro clínico estável, em ventilação espontânea ou com oxigenoterapia e que estivesse em internação a pelo menos 7 dias; foram dispensados aqueles que estavam em ventilação mecânica invasiva ou não, que estivesse em pré ou pós-operatório imediato, lesões na pele, doença neurológica, uso de sedativo ou que não tivesse prescrição de fisioterapia. A coleta contou com 15 participantes, sendo estes submetidos à terapia por 10 minutos com a água em temperatura de $37^{\circ} \mathrm{C}$, para avaliá-los os autores utilizaram as seguintes variáveis: FC, PAM (pressão arterial média) e SaO2 ea avaliação foi feita durante o período da manhã aproximadamente 7 horas.

No primeiro dia foi realizado avaliação, procedimento doloroso e reavaliação já no segundo dia, foi realizado o procedimento doloroso avaliação, fisioterapia aquática e reavaliação. O estudo obteve eficácia do tratamento para RNPT's especialmente no que se refere ao sono e FC no primeiro dia de atendimento, e de um modo geral a aplicação da técnica foi importante para FR reduzindo-a e parra SaO2, aumentando-a no segundo dia. 
Barbosa L, et al. (2015) intitulou sua pesquisa de "Impacto em recém-nascidos hospitalizados", sendo produzido no berçário do Hospital de Clinicas da Universidade Federal do Triangulo Mineiro, Ele contou com 10 pacientes que atendiam aos seguintes critérios de inclusão: estabilidade clínica, ventilação espontânea em ar ambiente. Foram excluídos aqueles que possuíam acesso venoso central ou periférico ou em processo infeccioso. Dos 10 pacientes que participaram, $60 \%$ eram do sexo masculino e $40 \%$ do sexo feminino, $80 \%$ estavam abaixo do peso, $30 \%$ foram gestações gemelares e $60 \%$ apresentavam quadro de DPOC (doença pulmonar obstrutiva crônica). O peso geral variou de 1330 a 2660 gramas e a idade gestacional entre 33 a 35 semanas. Os pacientes foram submetidos a terapia, porém os autores não informaram o tempo de duração da terapia, apenas a temperatura da água que era em torno de $36^{\circ}$ e $37^{\circ} \mathrm{C}$. Os autores realizam a recomendação da técnica para o manejo da dor de RNPT's hospitalizados, já que em sua pesquisa alcançaram sucesso, tanto no tratamento do quadro álgico, quanto na estabilização dos sinais vitais.

Vignochic C, et al. (2010) realizou uma pesquisa "intitulada efeitos da fisioterapia aquática na dor e no estado de sono vigília do recém-nascido pré termo estáveis na uti neonatal", realizada na UTI Neonatal do Hospital Luterano de Porto Alegre no estado Rio Grande do Sul, entre julho de 2005 e junho de 2007. Dos 12 participantes do estudo 6 eram do sexo feminino e 6 do sexo masculino, com idade gestacional inferior a 36 semanas. Para serem aceitos nos estudos, os pacientes deveriam ter comportamento anormal, intolerância ao toque, choro excessivo, sinais de dor, e agitação por mais de 60 minutos, além de estar clinicamente estável; dentro dos critérios de exclusão estavam: apresentar alteração da temperatura, alteração neurológica, infecção ou necessidade de suporte ventilatório. O procedimento durava cerca de 10 minutos, e a temperatura da água foi mantida em $37^{\circ} \mathrm{C}$, os pacientes eram enrolados em uma toalha, favorecendo a postura flexora, e então eram imersos na água. Os resultados foram muito positivos, com relação ao tratamento da dor.

Os autores indicam a terapia como tratamento não farmacológico da dor, por se mostrar muito efetivo também nos sinais vitais. Por fim, Novakoski K, et al. (2018) produziu um artigo chamado "De volta ao meio liquido: Efeitos da intervenção fisioterapêutica aquática realizada em prematuros", esse trabalho trata-se de um ensaio clínico feito na UTI neonatal do hospital público da Universidade do Paraná, composto por 22 pacientes, dos quais 14 eram do sexo feminino e 8 do sexo masculino e com idade gestacional corrigida no dia da intervenção de 30 a 37 semanas, com peso médio de 1858 gramas. Como critérios de inclusão os participantes deveriam estar estáveis clinicamente e com ventilação mecânica invasiva ou não, o único critério de exclusão usado foi em caso de acesso venoso central, o que impediria a imersão.

O procedimento teve duração de 10 minutos e a temperatura da água foi mantida entre $36,5^{\circ}$ e $37^{\circ} \mathrm{C}$. Os autores puderam comprovar a efetividade da técnica no sono e estado de vigília, além da melhora dos sinais vitais, e sem alteração da temperatura. De maneira geral, todos estudos apontaram um mesmo denominador comum, que foi a efetividade da técnica para o bem-estar do RNPT. Indiscutivelmente houve melhora dos sinais vitais evidenciando a redução do estresse melhorando, a qualidade de sono ganho e de peso.

Tabela 2 - Apresentação dos resultados encontrados pelos autores dos estudos supracitados.

\begin{tabular}{|c|c|c|c|}
\hline Autor/Ano & Variável & Antes do Procedimento & Depois do Procedimento \\
\hline \multirow{4}{*}{$\begin{array}{llll}\text { Barbosa } & \text { L, } & \text { et } & \text { al. } \\
(2015) & & & \end{array}$} & FC & 146 BPM & 129, 7 BPM \\
\hline & FR & 47,3 IR & $38,9 \mathrm{IR}$ \\
\hline & $\mathrm{SaO} 2$ & $94,6 \%$ & $97,5 \%$ \\
\hline & Dor & 3,6 NIPS & 0,3 NIPS \\
\hline \multirow{6}{*}{$\begin{array}{l}\text { Vigmochic C, et al. } \\
(2010)\end{array}$} & $\mathrm{FC}$ & 172,3 BPM & 147 BPM \\
\hline & FR & 54 IR & 44 IR \\
\hline & PAM & 56,8 & \\
\hline & TAX & $36,2^{\circ}$ & $36,5^{\circ}$ \\
\hline & $\mathrm{SaO} 2$ & $91 \%$ & $97,6 \%$ \\
\hline & DOR & 5,25 & 0,33 \\
\hline \multirow{2}{*}{$\begin{array}{l}\text { Novaskoski K, et al. } \\
(2018)\end{array}$} & FC & 154 BPM & 144BPM \\
\hline & $\mathrm{SaO} 2$ & $94,5 \%$ & $97,8 \%$ \\
\hline
\end{tabular}

Fonte: Brito RS, et al. (2020). 


\section{DISCUSSÃO}

Vignochi C, et al. (2010) em seu estudo relatou sobre a eficiência da ofurôterapia na diminuição da frequência cardíaca, comprovada através da resposta cardiológica positiva obtida através da técnica. Os autores puderam constatar esse efeito significativo nos pacientes da pesquisa, compreendendo assim o que Cunha M e Caromano F(2003) explicam em seu trabalho: pressão hidrostática exercida pela agua que realiza, o aprimoramento do retorno venoso, em cerca de $60 \%$, resultando de modo reflexivo na diminuição da frequência cardíaca. Rambo D e FilippinN (2019) em concordância realizou a indicação da ofuroterapia, pois também pode constatar em sua pesquisa a efetividade da técnica na redução da frequência cardíaca.

Vignochi C, et al. (2010) e Rambo D e Filippin N (2019) continuaram com um alinhamento de pensamento no que se refere a efetividade da técnica, e sobre a segurança que ela possui para os RNPT's hospitalizados, além de reafirmarem sua relevância como indicação de terapia analgésica não farmacológica no combate da sensorial da dor. Já que através da ação da água existe atenuação da capacidade de condução do estímulo da dor, reduzindo assim, o quadro álgico sofrido pelo RN (BARBOSA L, et al., 2015).

Barbosa L, et al. (2015) apresentou o sucesso da ofurôterapia em seu estudo em relação ao tratamento do quadro álgico sofrido pelos pacientes, onde em sua pesquisa obtiveram antes na intervenção terapêutica uma escala de dor que se encontrava em 3,1 e após a intervenção caiu para 0,3. Novakoski K, et al. (2018) e Rambo D e FilippinN (2019) tem absoluta concordância do uso da ofurôterapia como tratamento do quadro álgico, ambos em seus estudos testemunharam a ação da água aquecida viabilizando uma velocidade maior do estímulo para medula, inibindo as fibras responsáveis pela sensação dolorosa, de modo que há a estabilização do quadro álgico.

Barbosa L, et al. (2015) teve um posicionamento positivo quanto ao uso da técnica na melhora da frequência respiratória, o estudo afirma que técnica melhorou a oxigenação devido o favorecimento ao trabalho respiratório advindo da resistência exercida pela pressão hidrostática. Em contrapartida Silva $\mathrm{H}$, et al. (2017). Afirmou que apesar da ação da pressão hidrostática realizada sobre tórax e elevar o diafragma que aprimorando o trabalho respiratório, no estudo não foram obtidas alterações tão notáveis na frequência respiratória foi na saturação de oxigênio.

Ataíde V, et al. (2016) em seu relato de experiência apresentou a modalidade da ofurôterapia como um grande recurso para ganho de peso corporal para o RNPT. Silva H, et al. (2017) também é adepto desse pensamento, já que em seu estudo pode apontar a importância da técnica devido aos seus resultados, onde os pacientes submetidos a ofurôterapia apresentaram ganho de peso corporal relevante e contínuo ao longo das sessões. A eficiência da ofurôterapia no quesito ganho de peso corporal está pautada na ação fisiológica da água sobre a sensibilidade das terminações nervosas, aumento da capacidade vital, viabilizando o relaxamento muscular e a diminuição do cortisol (hormônio do estresse), essa ação causa o estado de repouso que de modo geral, faz com que haja redução do gasto energético, consequentemente, favorecendo o ganho de peso. (ATAÍDE V, et al., 2016)

Vignochic C, et al. (2010) discorreu a respeito do favorecimento que a ofurôterapia gerou na condução sono dos pacientes presentes na pesquisa, estes que anteriormente se encontravam agitados e em estado máximo de choro, como mensurado pelo estudo de Novakoski K, et al., (2018) tiveram pacientes, vigorosamente agitados e muito chorosos. Os autores supracitados alcançaram resultados significativos, seus pacientes regrediram em sua agitação, chegando repouso; os autores possuem um alinhamento quanto a recomendação da técnica, já que ela contribuiu na melhora da qualidade no sono. Tendo em vista que a terapia propícia um ambiente similar ao útero materno, através de uma experiência sensorial. Produzido pela turbulência, pressão hidrostática, empuxo e temperatura da água. Gerando conforto e facilitando assim que o RNPT encontre o aconchego que necessita para o estado de sono.

\section{CONSIDERAÇÕES FINAIS}

A terapia é de fácil manejo e, de baixo custo e tem potencial para melhorar a assistência ao paciente RNPT hospitalizado; de maneira que se torna enigmático o porquê de ainda não ser uma pratica difundida no Brasil, tendo em vista os benéficos que pode trazer a saúde dessas pacientes. Os estudos publicados a respeito do 
tema foram indispensáveis para construção desse trabalho, contudo há uma grande lacuna no material. É perceptível a necessidade de estudos mais aprofundados sobre a temática, tanto no meio acadêmico. Para que os acadêmicos e profissionais tenham acesso ao conhecimento da técnica, e desenvolvam da melhor forma possível suas condutas terapêuticas, quanto no meio social, para que população tenha acesso a esse recurso terapêutico que tem manifestado resultados tão assertivos.

\section{REFERÊNCIAS}

1. ATAÍDE V, et al. Ofurô em recém-nascidos pré-termo de baixo peso: relato de experiência. Assobrafir Ciência, 2016; $11 \mathrm{p}$.

2. BARBOSA L, et al. Impacto da hidroterpia em recém-nascidos hospitalizados. Fisioterapia Brasil, 2015.

3. CUNHA, M; CAROMANO, F. Efeitos fisiológicos da imersão e sua relação com privação sensorial e relaxamento em hidroterapia. Rev.Ter.ocup.univ, 2003.

4. GERHARDT T, SILVEIRA D. Métodos de pesquisa. E;UFRGS, 2009.

5. GONSALVESR, et al. Hidoterapia com ofurô como modalidade de fisioterpia no contexto hospitalar humanizado em neonatologia. Researhgate, 2017; p. 33.

6. MAIA, F. A fisioterpia nas unidade intensivas neonatal. Comunicações breves, 2015; p. 64-5.

7. MATTOS P. Tipos de literatura. P.2. Botucatu. 2015.

8. MINAYO M, et al. Pesquisa social. Petrópolis RJ: Vozes, 2009.

9. NOVAKOSKI K, et al. De volta ao meio liquido: efeitos da intervenção de fisioterapia aquática em prematuros. Rev. Bras.Cineantropom, 2018.

10. OLIVEIRA $\mathrm{C}$, et al. Perfil de récem-nascido pré-termo internado na unidade intensiva de hospital de alta complexidade. ABCS HEALTH SCIENCES, 2015; p. 5.

11. OLIVEIRA L, et al. Fatores maternos e neonatais relacionadosas a prematuridade. Revista escola de enfermagem da USP, 2016; p. 382-389.

12. PINHEIRO E, et al. Ruído na Unidade de Terapia Intensiva Neonatal e no interior da incubadora Latino-am enfermagem, 2011; p.19-8.

13. RAMBO D, FILIPPIN N. Efeitos da fisioterapia aquática em prematuros internados na unidade de terapia intensiva neonatal. $6^{\circ}$ congresso internacional de saúde cisaude, 2019; p.10-15.

14. RIBEIRO J, et al. O prematuro em unidades de terapia intensivas neonatal: a assitência do enfermeiro . Revista de enfermagem UFPE, p.9, abril 2016.

15. ROUT, A. Fisioterapia aquática para neonatal, $2000 ;$ p.8.

16. SALGE A, et al. Fatores matenos associados e neonatais associados a prematuridade. Revista eletrônica de enfermagem,2009; p. 642-646.

17. SILVA H. Efeitos fisiológicos da hidroterapia em balde em recém-nascidos prematuros. Terapia ocupacional-USP, 2017; p.7.

18. TANTERFFER A, et al. Fisioterapia aquática para neonatos. Vitrine de produção acadêmica II faculdade Dom Bosco, s.d; p. 83-94.

19. VIGNOCHIC C, et al. Efeitos da fisioterapia aquática na dor e no estado de sono vigília de recém-nascidos pre-termo estáveis internados na unidade de terapia intensiva neonatal. Revista. Brasileira de fisioterapia, 2010; p. $214-220$. 